\title{
Fault Detection for Discrete-Time Nonlinear Impulsive Switched Systems
}

\author{
Qingyu Su, Xiaolong Jia, and Zhengfan Song \\ School of Automation Engineering, Northeast Dianli University, Jilin, Jilin Province 132012, China \\ Correspondence should be addressed to Qingyu Su; suqingyuphd@yeah.net
}

Received 9 April 2015; Revised 25 June 2015; Accepted 2 July 2015

Academic Editor: Elena Litsyn

Copyright (C) 2015 Qingyu Su et al. This is an open access article distributed under the Creative Commons Attribution License, which permits unrestricted use, distribution, and reproduction in any medium, provided the original work is properly cited.

\begin{abstract}
This paper investigates the fault detection problem for discrete-time nonlinear impulsive switched systems. Attention is focused on designing the fault detection filters to guarantee the robust performance and the detection performance. Based on these performances, sufficient conditions for the existence of filters are given in the framework of linear matrix inequality; furthermore, the filter gains are characterized by a convex optimization problem. The presented technique is validated by an example. Simulation results indicate that the proposed method can effectively detect the faults.
\end{abstract}

\section{Introduction}

Fault detection (FD) is an important topic in system engineering from the viewpoint of the higher demands for safety and reliability of control systems [1,2]. The basic idea of the model-based FD is to design observers [3] or filters [4] and generate an residual signal. Since the value counted by the residual evaluation function is larger than the predefined threshold, an alarm is generated. To date, there are many methods to solve the FD problem. As one of the typical methods, the FD problem is converted into a robust filtering problem; then $H_{\infty}$ technique is presented $[5,6]$. For another method, FD systems have been directly considered to be sensitive to the faults and simultaneously robust to the unknown disturbance, then the $H_{\infty} / H_{-}$technique investigates this important issue $[7,8]$.

On the other hand, switched systems which belong to hybrid systems consist of a finite number of subsystems and a logical rule that orchestrates switching between these subsystems [9]. The primary motivation for studying switched systems comes partly from the fact that switched systems and switched multicontroller systems have numerous applications in control of flight control [10], missile autopilot design [11], chemical systems [12], networked control systems [13], and many other fields. Until now, a number of recent results are focused on stability and stabilizability under arbitrary switching [14], restricted switching (like dwell time and average dwell time $[15,16]$ ), multiple Lyapunov functions method, and piecewise quadratic Lyapunov functions. As one of the special switched systems, impulsive switched systems produce impulses when the system is switching among subsystems, and There is also a wide range of actual systems such as engineering, economics, and biology. This kind of impulses will cause instability and oscillations and lead to poor performance. Recently, a number of papers have focused on stability problem of the impulsive switched systems [1721].

However, the problem of FD design in switched systems schemes is still in the early stage of development and a few results have been reported in the literatures [22-26]. To the best of the authors' knowledge, the FD problem for impulsive switched systems, especially discrete-time nonlinear impulsive switched systems, has not been investigated yet. It is worth noting that the FD approaches for switched systems without impulses are not appropriate for switched systems with impulses due to the effect of the impulse in switching point. Therefore, a new FD technique is needed to solve the impulsive case. Moreover, even if the mathematics model for the actual system are established with neglecting the impulse in switching point, the inaccurate mathematics model may reduce the robustness of the actual system, and the results may increase the risk of the false alarm. Thus, it is 
necessary to directly investigate the fault detection problem for impulsive switched systems. As the significance in theory and practice, the FD problem for discrete-time nonlinear impulsive switched systems should be investigated, which motivates us to study this interesting issue.

In this paper, we consider a general class nonlinear impulsive switched system with nonlinear impulsive increments, and the fault detection problem for this class of systems is investigated. Firstly, a weighted $l_{2}$ performance for discrete-time nonlinear impulsive switched systems is presented; meanwhile, the $H_{-}$performance of discrete-time nonlinear impulsive switched systems is derived to reflect the effect on the residual signal from the faults. Subsequently, sufficient conditions for the weighted $l_{2}$ performance and the $H_{-}$performance are formulated by linear matrix inequalities (LMIs) with less conservatism. Finally, the filters gains are characterized in terms of the solution of a convex optimization problem.

The paper is organized as follows. Section 2 introduces the problem under consideration and presents the design objectives. Section 3 illustrates the FD filter design approach in detail. An example is given in Section 4 to demonstrate the proposed method. Conclusions of this paper are given in the last section.

Notation. The superscripts $T,-1$ stand for the transposition and the inverse of a matrix, respectively. The matrices $A>$ $0(A \geq 0)$ and $A<0(A \leq 0)$ denote positive-definiteness (positive semidefinite matrix) and negative-definiteness (negative semidefinite matrix). I and 0 represent the identity matrix and the zero matrix with appropriate dimensions, respectively. The Hermitian part of a square matrix $M$ is denoted by $\operatorname{He}(M):=M+M^{T}$. The symbol $*$ within a matrix represents the symmetric entries.

\section{Problem Formulation}

2.1. System Model. Consider the following discrete-time nonlinear impulsive switched systems:

$$
\begin{aligned}
& x(k+1)=\sum_{j=1}^{N} \xi_{j}(k) \\
& \cdot\left(A_{j} x(k)+B_{j 1} d(k)+B_{j 2} f(k)+\Upsilon_{j}(k, x(k))\right), \\
& \Delta x(k)=\sum_{j=1}^{N} \xi_{j}(k)\left(H_{j} x(k)+\Omega_{j}(k, x(k))\right), \quad k=k_{i}, \\
& y(k)=\sum_{j=1}^{N} \xi_{j}(k)\left(C_{j} x(k)+D_{j 1} d(k)+D_{j 2} f(k)\right), \\
& x\left(k_{0}^{+}\right)=x_{0},
\end{aligned}
$$

where $x(k) \in R^{n}$ is the state and $y(k) \in R^{m}$ is the measured output. $d(k) \in R^{n_{d}}$ and $f(k) \in R^{n_{f}}$ are the disturbance input and the fault, respectively, which are energy bounded; then they are demanded to belong to $l_{2}\left[\begin{array}{ll}0 & \infty\end{array}\right)$. $k_{i}, i=0,1,2, \ldots$, is impulsive switching time points. Denote $\mathcal{N}=\left\{\begin{array}{lll}1 & \cdots & N\end{array}\right\}$. The switching signal $\xi_{j}(k): Z^{+} \rightarrow\{0,1\}$ specifies that $j$ th subsystem is activated when $\xi_{j}(k)=1$, and $\sum_{j=1}^{N} \xi_{j}(k)=1 . \Upsilon_{j}(k, x(k)):\left[k_{0}, \infty\right) \times R^{n} \rightarrow R^{n}$, which is globally Lipschitz continuous, and $\Upsilon_{j}(k, 0) \equiv 0$ for all $k \in\left[k_{0}, \infty\right) \Delta x\left(k_{i}\right)=x\left(k_{i}^{+}\right)-x\left(k_{i}^{-}\right)=x\left(k_{i}^{+}\right)-$ $x\left(k_{i}\right)$, with $x\left(k_{i}^{+}\right)=\lim _{k \rightarrow k_{i}^{+}} x(k)$ and $x\left(k_{i}\right)=x\left(k_{i}^{-}\right)=$ $\lim _{k \rightarrow k_{i}^{-}} x(k)$; that is, the solution $x(t)$ is left continuous. $\Omega_{j}(k, x(k)):\left[k_{0}, \infty\right) \times R^{n} \rightarrow R^{n}$ is nonlinear functions, and $\Omega_{k}(k, 0) \equiv 0$ for all $t \in\left[k_{0}, \infty\right)$. The $j$ th subsystem is denoted by the matrices $A_{j}, B_{j 1}, B_{j 2}, H_{j}, C_{j}, D_{j 1}$, and $D_{j 2}$ with appropriate dimensions.

The following assumptions for nonlinear impulsive switched system (1) are introduced.

Assumption 1. There exist nonnegative scalars $g_{j}>0$, such that

$$
\Upsilon_{j}^{T}(k, x(k)) \Upsilon_{j}(k, x(k)) \leq g_{j} x^{T}(k) x(k), \quad j \in \mathcal{N} .
$$

Assumption 2. Denote by $\rho(\cdot)$ the spectral radius for each subsystem and $\rho_{h}=\max _{j \in \mathcal{N}} \rho\left(H_{j}+I\right)$; then $\left\|\Omega_{j}\left(k_{i}, x\left(k_{i}\right)\right)\right\| \leq$ $\rho_{h}\left\|x\left(k_{i}\right)\right\|$.

Remark 3. Note that owing to the presence of system nonlinearity, the above assumptions essentially draw from the analysis of the stability for the system. Moreover, they are two basic conditions for this kind of systems (see [27] for further discussion). Therefore we consider the nonlinear impulsive switched systems, which satisfies Assumptions 1 and 2.

For the purpose of the fault detection, the following FD filters are designed:

$$
\begin{array}{r}
x_{f}(k+1)=\sum_{j=1}^{N} \xi_{j}(k)\left(A_{f j} x_{f}(k)+B_{f j} y(k)\right), \\
r(k)=\sum_{j=1}^{N} \xi_{j}(k)\left(C_{f j} x_{f}(k)+D_{f j} y(k)\right),
\end{array}
$$

where $x_{f}(k)$ is the state of the filter, $r(k)$ is the residual signal, and $j \in \mathcal{N}$. The matrices $A_{f j}, B_{f j}, C_{f j}$, and $D_{f j}$ with appropriate dimensions are to be determined.

Denoting the augmented state vector $\tilde{x}(k)=\left[x(k)^{T}\right.$, $\left.x_{f}(k)^{T}\right]^{T}$ and augmenting the model of system (1) to include the states of (3), we can obtain the augmented system as follows:

$$
\begin{aligned}
& \tilde{x}(k+1)=\sum_{j=1}^{N} \xi_{j}(k)\left(\mathscr{A}_{j} \tilde{x}(k)+\mathscr{B}_{j 1} d(k)+\mathscr{B}_{j 2} f(k)\right. \\
& \left.\quad+\widetilde{\Upsilon}_{j}(k, \tilde{x}(k))\right), \quad k \neq k_{i}, \\
& \Delta \widetilde{x}(k)=\sum_{j=1}^{N} \xi_{j}(k)\left(\mathscr{H}_{j} x(k)+\widetilde{\Omega}_{j}(k, \tilde{x}(k))\right), \quad k=k_{i},
\end{aligned}
$$




$$
\begin{aligned}
& r(k)=\sum_{j=1}^{N} \xi_{j}(k)\left(\mathscr{C}_{j} \tilde{x}(k)+\mathscr{D}_{j 1} d(k)+\mathscr{D}_{j 2} f(k)\right), \\
& \tilde{x}\left(k_{0}^{+}\right)=\tilde{x}_{0},
\end{aligned}
$$

where $j \in \mathcal{N}$,

$$
\begin{aligned}
\mathscr{A}_{j} & =\left[\begin{array}{cc}
A_{j} & 0 \\
B_{f j} C_{j} & A_{f j}
\end{array}\right], \\
\mathscr{B}_{j 1} & =\left[\begin{array}{c}
B_{j 1} \\
B_{f j} D_{j 1}
\end{array}\right], \\
\mathscr{B}_{j 2} & =\left[\begin{array}{c}
B_{j 2} \\
B_{f j} D_{j 2}
\end{array}\right], \\
\mathscr{H}_{j} & =\left[\begin{array}{cc}
H_{j} & 0 \\
0 & 0
\end{array}\right], \\
\widetilde{\Upsilon}_{j}(k, \tilde{x}(k)) & =\left[\begin{array}{c}
\Upsilon_{j}(k, x(k)) \\
0
\end{array}\right], \\
\widetilde{\Omega}_{j}(k, \widetilde{x}(k)) & =\left[\begin{array}{c}
\Omega_{j}(k, x(k)) \\
0
\end{array}\right], \\
\mathscr{C}_{j} & =\left[\begin{array}{ll}
D_{f j} C_{j} & C_{f j}
\end{array}\right], \\
\mathscr{D}_{j 1} & =D_{f j} D_{j 1}, \\
\mathscr{D}_{j 2} & =D_{f j} D_{j 2} .
\end{aligned}
$$

To present the purpose of this paper more precisely, the following definition is introduced.

Definition 4. Let Assumptions 1 and 2 be satisfied and $d(k)=$ 0 . Nonlinear impulsive switched system (4) under zero-initial conditions is said to be stable with the $H_{-}$-gain $\beta$, if the condition holds that

$$
\sum_{k=0}^{\infty} r(k)^{T} r(k) \geq \beta^{2} \sum_{k=0}^{\infty} f(k)^{T} f(k) .
$$

2.2. Problem Formulation. The Frameworks of FD Filter Design. Given nonlinear impulsive switched system (1), the FD filters (3) are designed such that nonlinear impulsive switched system (4) is stable, and the fault effects on the residual signal are maximized, while the disturbance effects on the residual signals are minimized. Our design objective of the FD filters can be formulated as the following performances:

$$
\begin{aligned}
\sum_{k=0}^{\infty}(1-\alpha)^{k} r(k)^{T} r(k) & \leq \gamma^{2} \sum_{k=0}^{\infty} d(k)^{T} d(k), \\
\sum_{k=0}^{\infty} r(k)^{T} r(k) & \geq \beta^{2} \sum_{k=0}^{\infty} f(k)^{T} f(k) .
\end{aligned}
$$

Remark 5. Condition (7) is used for the disturbance attenuation performance, which minimizes the disturbance effects on the residual output and ensures that the disturbance is not disastrous. Condition (8) is expressed to maximize the effects of the fault $f(k)$ on the residual output $r(k)$. That is, the residual output $r(k)$ is sensitive to the fault $f(k)$.

After designing the residual generator, how to evaluate the generated residual is considered. One of the widely adopted approaches is to select an appropriate threshold and an appropriate residual evaluation function. Similar to that proposed in [28], the residual evaluation function $J_{r(k)}(k)$ can be chosen as

$$
J_{r(k)}(k)=\sqrt{\frac{1}{k} \sum_{s=1}^{k} r^{T}(s) r(s)},
$$

where $k$ denotes the evaluation time step.

Let $J_{\text {th }}=\sup _{d(k) \in l_{2}, f(k)=0} J_{r(k)}(k)$ be the threshold. Based on this, the occurrence of faults can be detected by comparing $J_{r(k)}(k)$ and $J_{\text {th }}$ according to the following logical relationship:

$$
\begin{aligned}
& \left\|J_{r(k)}\right\| \leq J_{\text {th }} \Longrightarrow \text { no fault } \Longrightarrow \text { no alarm, } \\
& \left\|J_{r(k)}\right\|>J_{\text {th }} \Longrightarrow \text { fault } \Longrightarrow \text { alarm. }
\end{aligned}
$$

\section{The Fault Detection Filter Design}

Before beginning this section, the following lemmas are needed to present our main results.

Lemma 6 (see [27]). Let $\epsilon>0$ be a given scalar and $\Xi \in R^{p \times q}$ a matrix such that $\Xi^{T} \Xi \leq I$, where $I$ is an identity matrix with appropriate dimension. Then $2 x^{T} \Xi y \leq \epsilon x^{T} x+\epsilon^{-1} y^{T} y$ for all $x \in R^{p}$ and $y \in R^{q}$.

Lemma 7 (see [29]). Let $P \in R^{n \times n}$ be a given symmetric positive definite matrix and let $Q \in R^{n \times n}$ be a given symmetric matrix. Then $\lambda_{\min }\left\{P^{-1} Q\right\} x(t)^{T} P x(t) \leq x(t)^{T} Q x(t) \leq$ $\lambda_{\max }\left\{P^{-1} Q\right\} x(t)^{T} P x(t)$ for all $x(t) \in R^{n}$, while $\lambda_{\max }\{\cdot\}$ and $\lambda_{\min }\{\cdot\}$ denote, respectively, the largest and the smallest eigenvalues of the matrix inside the brackets.

In this section, sufficient conditions on the existence of the FD filters for nonlinear impulsive switched systems (4) would be given, and the desired filter gains can be obtained.

3.1. The Disturbance Attenuation Performance (8). Considering discrete-time nonlinear impulsive switched system (4) with $f(k)=0$, we have

$$
\begin{array}{r}
\tilde{x}(k+1) \\
=\sum_{j=1}^{N} \xi_{j}(k)\left(\mathscr{A}_{j} \tilde{x}(k)+\mathscr{B}_{j 1} d(k)+\widetilde{Y}_{j}(k, \tilde{x}(k))\right), \\
k \neq k_{i},
\end{array}
$$




$$
\begin{aligned}
& \Delta \tilde{x}(k)=\sum_{j=1}^{N} \xi_{j}(k)\left(\mathscr{H}_{j} x(k)+\widetilde{\Omega}_{j}(k, \tilde{x}(k))\right), \quad k=k_{i}, \\
& r(k)=\sum_{j=1}^{N} \xi_{j}(k)\left(\mathscr{C}_{j} \tilde{x}(k)+\mathscr{D}_{j 1} d(k)\right), \\
& \tilde{x}\left(k_{0}^{+}\right)=\tilde{x}_{0} .
\end{aligned}
$$

Firstly, the weighted $l_{2}$ performance for nonlinear impulsive switched system (11) is given.

Lemma 8. Let $\alpha, \varepsilon_{j 1}, \varepsilon_{j 2}$, and $\varepsilon_{j 3}$ be constants satisfying $0<$ $\alpha<1, \varepsilon_{j 1}>0, \varepsilon_{j 2}>0$, and $\varepsilon_{j 3}>0$, and Assumptions 1 and 2 hold. Furthermore, suppose that discrete-time nonlinear impulsive switched system (11) switches from pth subsystem to $j$ th subsystem as switched time point $k_{i}$. If there exist $\lambda_{j}>$ 0 and Lyapunov functions candidate $V_{j}(k)=\tilde{x}(k)^{T} \mathscr{P}_{j} \tilde{x}(k)$ satisfying

$$
\begin{aligned}
& 0 \leq \mathscr{P}_{j} \leq \lambda_{j} I, \\
& {\left[\begin{array}{cc}
\Theta_{j} & \mathscr{A}_{j}^{T} \mathscr{P}_{j} \mathscr{B}_{j 1}+\mathscr{C}_{j}^{T} \mathscr{D}_{j 1} \\
* & -\gamma^{2} I+\mathscr{B}_{j 1}^{T}\left(\mathscr{P}_{j}+\varepsilon_{j 2}^{-1} \mathscr{P}_{j}^{2}\right) \mathscr{B}_{j 1}+\mathscr{D}_{j 1}^{T} \mathscr{D}_{j 1}
\end{array}\right]} \\
& \quad<0, \quad j \in \mathscr{N},
\end{aligned}
$$

where $\Theta_{j}=\mathscr{A}_{j}^{T}\left(\mathscr{P}_{j}+\varepsilon_{j 1}^{-1} \mathscr{P}_{j}^{2}\right) \mathscr{A}_{j}-(1-\alpha) \mathscr{P}_{j}+\left(\varepsilon_{j 1}+\varepsilon_{j 2}+\lambda_{j}\right) g_{j} I+$ $\mathscr{C}_{j}^{T} \mathscr{C}_{j}$, then nonlinear impulsive switched system (11) is stable with the weight $l_{2}$-gain $\gamma$ for any switching signal satisfying

$$
\tau_{p} \geq \operatorname{ceil}\left[-\frac{\ln \mu_{p j}}{\ln (1-\alpha)}\right]
$$

where $\mu_{p j}=\left(\left(\varepsilon_{j 3}+1\right) \lambda_{\max }\left\{\left(\mathscr{H}_{j}+I\right)^{T} \mathscr{P}_{j}\left(\mathscr{H}_{j}+I\right)\right\}+\left(\varepsilon_{j 3}^{-1}+\right.\right.$ 1) $\left.\rho_{h}^{2} \lambda_{\max }\left\{\mathscr{P}_{j}\right\}\right) / \lambda_{\min }\left\{\mathscr{P}_{p}\right\}, p, j \in \mathcal{N}$, and the function ceil $(\nu)$ represents rounding real number $\nu$ to the nearest integer greater than or equal to $\nu$.

Proof. See the appendices.

Subsequently, inequality conditions for the disturbance attenuation performance (8) are constructed.

Theorem 9. Let $\gamma, \alpha, \varepsilon_{j 1}$, and $\varepsilon_{j 2}$ be constants satisfying $\gamma>0$, $0<\alpha<1, \varepsilon_{j 1}>0$, and $\varepsilon_{j 2}>0$. If there exist matrix variables $\widehat{A}_{f j}, \widehat{B}_{f j}, \widehat{C}_{f j}, \widehat{D}_{f j}, R_{j}$, and $\lambda_{j}$ and symmetric positivedefinite matrices

$$
\mathscr{P}_{j}=\left[\begin{array}{cc}
\mathscr{P}_{j 1} & \mathscr{P}_{j 2} \\
* & \mathscr{P}_{j 2}
\end{array}\right]>0, \quad j \in \mathcal{N},
$$

satisfying the following inequalities:

$$
\begin{aligned}
& 0 \leq \mathscr{P}_{j} \leq \lambda_{j} I, \\
& {\left[\begin{array}{cccccc}
-\bar{\alpha} \mathscr{P}_{j}+\varphi_{a j} I & 0 & \Xi_{a 13} & \Xi_{a 14} & 0 & \Xi_{a 16} \\
* & -\gamma^{2} I & \Xi_{a 23} & 0 & \Xi_{a 25} & D_{j 1}^{T} \widehat{D}_{f j}^{T} \\
* & * & -\mathscr{P}_{j} & 0 & 0 & 0 \\
* & * & * & -\varepsilon_{j 1} I & 0 & 0 \\
* & * & * & * & -\varepsilon_{j 2} I & 0 \\
* & * & * & * & 0 & I-\operatorname{He}\left(R_{j}\right)
\end{array}\right]}
\end{aligned}
$$

$<0$,

where $\bar{\alpha}=1-\alpha, \varphi_{a j}=\left(\varepsilon_{j 1}+\varepsilon_{j 2}+\lambda_{j}\right) g_{j}$,

$$
\begin{aligned}
& \Xi_{a 13}=\Xi_{a 14}=\left[\begin{array}{cc}
A_{j}^{T} \mathscr{P}_{j 1}+C_{j}^{T} \widehat{B}_{f j}^{T} & A_{j}^{T} \mathscr{P}_{j 2}+C_{j}^{T} \widehat{B}_{f j}^{T} \\
\widehat{A}_{f j}^{T} & \widehat{A}_{f j}^{T}
\end{array}\right], \\
& \Xi_{a 23}=\Xi_{a 25}=\left[\begin{array}{ll}
B_{j 1}^{T} \mathscr{P}_{j 1}+D_{j 1}^{T} \widehat{B}_{f j}^{T} & B_{j 1}^{T} \mathscr{P}_{j 2}+D_{j 1}^{T} \widehat{B}_{f j}^{T}
\end{array}\right], \\
& \Xi_{a 16}=\left[\begin{array}{c}
C_{j}^{T} \widehat{D}_{f j}^{T} \\
\widehat{C}_{f j}^{T}
\end{array}\right],
\end{aligned}
$$

then switched system (11) is asymptotically stable for any switching signal satisfying (14) and guarantees the weighted $l_{2}$ performance $\sum_{k=0}^{\infty}(1-\alpha)^{k} r(k)^{T} r(k) \leq \gamma^{2} \sum_{k=0}^{\infty} d(k)^{T} d(k)$.

Proof. See the appendices.

3.2. The Fault Sensitiveness Performance (8). Considering discrete-time nonlinear impulsive switched system (4) with $d(k)=0$, we have

$$
\begin{aligned}
& \tilde{x}(k+1) \\
& =\sum_{j=1}^{N} \xi_{j}(k)\left(\mathscr{A}_{j} \tilde{x}(k)+\mathscr{B}_{j 2} f(k)+\widetilde{Y}_{j}(k, \tilde{x}(k))\right), \\
& \Delta \tilde{x}(k)=\sum_{j=1}^{N} \xi_{j}(k)\left(\mathscr{H}_{j} x(k)+\widetilde{\Omega}_{j}(k, \tilde{x}(k))\right), \quad k=k_{i}, \\
& r(k)=\sum_{j=1}^{N} \xi_{j}(k)\left(\mathscr{C}_{j} \tilde{x}(k)+\mathscr{D}_{j 2} f(k)\right), \\
& \tilde{x}\left(k_{0}^{+}\right)=\tilde{x}_{0} .
\end{aligned}
$$

$H_{-}$performance for discrete-time nonlinear impulsive switched system (19) is given.

Lemma 10. Let $\alpha, \varepsilon_{j 1}$, and $\varepsilon_{j 4}$ be constants satisfying $0<$ $\alpha<1, \varepsilon_{j 1}>0$, and $\varepsilon_{j 4}>0$, and Assumptions 1 and 2 hold. Furthermore, suppose that nonlinear impulsive switched system (19) switches from pth subsystem to jth subsystem as 
switched time point $k_{i}$. If there exist $\lambda_{j}>0$ and Lyapunov functions candidate $V_{j}(k)=\tilde{x}(k)^{T} \mathscr{P}_{j} \tilde{x}(k)$ satisfying

$$
\begin{aligned}
& 0 \leq \mathscr{P}_{j} \leq \lambda_{j} I, \\
& {\left[\begin{array}{cc}
\Phi j & \mathscr{B}_{j 2}^{T} \mathscr{P}_{j} \mathscr{A}_{j}-\mathscr{C}_{j}^{T} \mathscr{D}_{j 2} \\
* & \beta^{2} I+\mathscr{B}_{j 2}^{T}\left(\mathscr{P}_{j}+\varepsilon_{j 4}^{-1} \mathscr{P}_{j}^{2}\right) \mathscr{B}_{j 2}-\mathscr{D}_{j 2}^{T} \mathscr{D}_{j 2}
\end{array}\right]} \\
& \quad<0, \quad j \in \mathcal{N},
\end{aligned}
$$

where $\Phi j=\mathscr{A}_{j}^{T}\left(\mathscr{P}_{j}+\varepsilon_{j 1}^{-1} \mathscr{P}_{j}^{2}\right) \mathscr{A}_{j}-(1-\alpha) \mathscr{P}_{j}+\left(\varepsilon_{j 1}+\varepsilon_{j 4}+\lambda_{j}\right) g_{j} I-$ $\mathscr{C}_{j}^{T} \mathscr{C}_{j}$, then discrete-time nonlinear impulsive switched system (19) is stable with the $H_{-}$-gain $\beta$ for any switching signal satisfying (14).

Proof. See the appendices.

Based on Lemma 10, the following theorem is given to obtain sufficient conditions by linear matrix inequalities.

Theorem 11. Let $\beta, \alpha, \varepsilon_{j 1}$, and $\varepsilon_{j 4}$ be constants satisfying $\beta>0$, $0<\alpha<1, \varepsilon_{j 1}>0$, and $\varepsilon_{j 4}>0$. If there exist matrix variables $\widehat{A}_{f j}, \widehat{B}_{f j}, \widehat{C}_{f f j}=\left[\widehat{C}_{f j} 0\right], \widehat{D}_{f f j}=\left[\widehat{D}_{f j} 0\right], \mathscr{R}_{j}=\left[R_{j}, 0\right]$, and $\lambda_{j}$ and symmetric positive-definite matrices

$$
\mathscr{P}_{j}=\left[\begin{array}{cc}
\mathscr{P}_{j 1} & \mathscr{P}_{j 2} \\
* & \mathscr{P}_{j 2}
\end{array}\right]>0, \quad j \in \mathcal{N},
$$

satisfying the following inequalities:

$$
\begin{aligned}
& 0 \leq \mathscr{P}_{j} \leq \lambda_{j} I, \\
& {\left[\begin{array}{cccccc}
-I & \Xi_{b 12} & -R_{j} & 0 & 0 & 0 \\
* & \Xi_{b 22}+\varphi_{b j} I & \Xi_{b 23} & \Xi_{b 24} & \Xi_{b 25} & 0 \\
* & * & \beta^{2} I+\operatorname{He}\left(D_{j 2}^{T} \widehat{D}_{f j}\right) & \Xi_{b 34} & 0 & \Xi_{b 36} \\
* & * & * & -\mathscr{P}_{j} & 0 & 0 \\
* & * & * & * & -\varepsilon_{j 1} I & 0 \\
* & * & * & * & * & -\varepsilon_{j 4} I
\end{array}\right]} \\
& <0,
\end{aligned}
$$

where $\bar{\alpha}=1-\alpha, \varphi_{b j}=\left(\varepsilon_{j 1}+\varepsilon_{j 4}+\lambda_{j}\right) g_{j}$,

$$
\begin{aligned}
& \Xi_{b 12}=\left[\begin{array}{ll}
-\mathscr{R}_{j} & -\mathscr{R}_{j}
\end{array}\right], \\
& \Xi_{b 22} \\
& =\left[\begin{array}{cc}
-\bar{\alpha} \mathscr{P}_{j 1}+\operatorname{He}\left(C_{j}^{T} \widehat{D}_{f f j}^{T}\right) & -\bar{\alpha} \mathscr{P}_{j 2}+C_{j}^{T} \widehat{D}_{f f j}^{T}+\widehat{C}_{f f j} \\
* & -\bar{\alpha} \mathscr{P}_{j 2}+\operatorname{He}\left(\widehat{C}_{f f j}^{T}\right)
\end{array}\right], \\
& \Xi_{b 23}=\left[\begin{array}{cc}
C_{j}^{T} \widehat{D}_{f j}^{T}+\widehat{D}_{f f j} D_{j 2} \\
\widehat{C}_{f j}^{T}+\widehat{D}_{f f j} D_{j 2}
\end{array}\right],
\end{aligned}
$$

$$
\begin{aligned}
& \Xi_{b 24}=\Xi_{b 25}=\left[\begin{array}{cc}
A_{j}^{T} \mathscr{P}_{j 1}+C_{j}^{T} \widehat{B}_{f j}^{T} & A_{j}^{T} \mathscr{P}_{j 2}+C_{j}^{T} \widehat{B}_{f j}^{T} \\
\widehat{A}_{f j}^{T} & \widehat{A}_{f j}^{T}
\end{array}\right], \\
& \Xi_{b 34}=\Xi_{b 36}=\left[B_{j 2}^{T} \mathscr{P}_{j 1}+D_{j 2}^{T} \widehat{B}_{f j}^{T} B_{j 2}^{T} \mathscr{P}_{j 2}+D_{j 2}^{T} \widehat{B}_{f j}^{T}\right] \text {, }
\end{aligned}
$$

then discrete-time nonlinear impulsive switched system (19) is asymptotically stable for any switching signal satisfying (14) and guarantees $H_{-}$performance:

$$
\sum_{k=0}^{\infty} \beta^{2} f(k)^{T} f(k) \leq \sum_{k=0}^{\infty} r(k)^{T} r(k) .
$$

Proof. See the appendices.

3.3. Algorithm. In the previous sections, Theorems 9 and 11 have formulated the inequality conditions for the performances (7) and (8), respectively. Summarily, we have the following algorithm.

It is noted that conditions (16), (17), (23), and (24) are all convex. Hence, the problem of FD filter design can directly translate into the following optimization problem:

$$
\begin{array}{ll}
\max & \beta, \\
\text { s.t. } & (16),(17),(23) \text { and }(24), \quad j \in \mathcal{N} .
\end{array}
$$

Moreover, if (27) is feasible, then the FD filter gains can be given by

$$
\left[\begin{array}{cc}
A_{f j} & B_{f j} \\
C_{f j} & D_{f j}
\end{array}\right]=\left[\begin{array}{cc}
\mathscr{P}_{j 2} & 0 \\
0 & R_{j}^{T}
\end{array}\right]^{-1}\left[\begin{array}{cc}
\widehat{A}_{f j} & \widehat{B}_{f j} \\
\widehat{C}_{f j} & \widehat{D}_{f j}
\end{array}\right] .
$$

\section{Examples}

In this section, we present a numerical example to illustrate the effectiveness of FD design approach. Consider the discrete-time nonlinear impulsive switched systems (1) with two subsystems and two parameters:

$$
\begin{aligned}
A_{1} & =\left[\begin{array}{ll}
-0.12 & 0.53 \\
-0.23 & 0.59
\end{array}\right], \\
A_{2} & =\left[\begin{array}{cc}
-0.60 & 0.23 \\
-0.49 & -0.05
\end{array}\right], \\
B_{11} & =B_{21}=\left[\begin{array}{c}
-0.17 \\
-0.16
\end{array}\right], \\
B_{12} & =B_{22}=\left[\begin{array}{c}
-0.14 \\
0.05
\end{array}\right], \\
\Omega_{1}\left(k_{i}, x\left(k_{i}\right)\right) & =\Omega_{2}(t, x(t))=\left[\begin{array}{c}
0.2 \sin \left(x_{1}\left(k_{i}\right)\right) \\
0.2 \sin \left(x_{2}\left(k_{i}\right)\right)
\end{array}\right], \\
\Upsilon_{1}(k, x(k)) & =\left[\begin{array}{c}
0.1 \sin \left(x_{1}(k)\right) e^{-0.5 k} \\
0
\end{array}\right],
\end{aligned}
$$




$$
\begin{aligned}
\Upsilon_{2}(k, x(k)) & =\left[\begin{array}{c}
0 \\
0.1 \sin \left(x_{2}(k)\right) e^{-0.5 k}
\end{array}\right], \\
H_{1} & =H_{2}=\left[\begin{array}{cc}
0.3 & 0 \\
0 & 0.3
\end{array}\right], \\
C_{1} & =C_{2}=\left[\begin{array}{ll}
0.3 & -0.1
\end{array}\right] \\
D_{11} & =D_{21}=0.1
\end{aligned}
$$

Given $\gamma=1, \alpha_{j}=0.4$, and $\varepsilon_{j 1}=\varepsilon_{j 2}=\varepsilon_{j 3}=\varepsilon_{j 4}=1$ and choosing $g_{j}=1, \rho_{j}=0.32$, we solve the convex optimization problem (27) and get the optimal sensitivity performance gain $\beta=0.3561$. The gain matrices of fault detection filters and matrix in Lyapunov functions are obtained as

$$
\begin{aligned}
& A_{f 1}=\left[\begin{array}{cc}
0.0664 & 0.2438 \\
-0.1053 & 0.3415
\end{array}\right] \text {, } \\
& B_{f 1}=\left[\begin{array}{c}
0.6063 \\
-0.2671
\end{array}\right] \text {, } \\
& C_{f 1}=\left[\begin{array}{ll}
-0.4074 & 0.0660
\end{array}\right] \text {, } \\
& D_{f 1}=-2.0153 \text {, } \\
& A_{f 2}=\left[\begin{array}{cc}
-0.2878 & 0.1567 \\
-0.3807 & -0.0119
\end{array}\right] \text {, } \\
& B_{f 2}=\left[\begin{array}{l}
1.0665 \\
0.0108
\end{array}\right] \text {, } \\
& C_{f 2}=\left[\begin{array}{ll}
-0.4265 & 0.0314
\end{array}\right] \text {, } \\
& D_{f 2}=-2.1530 \text {, } \\
& \mathscr{P}_{1}=\left[\begin{array}{cccc}
1.5526 & -1.0070 & 0.6676 & -0.2132 \\
-1.0070 & 1.8900 & -0.2132 & 0.4465 \\
0.6676 & -0.2132 & 0.6676 & -0.2132 \\
-0.2132 & 0.4465 & -0.2132 & 0.4465
\end{array}\right], \\
& \mathscr{P}_{2}=\left[\begin{array}{cccc}
2.5529 & -1.0351 & 1.1314 & -0.3521 \\
-1.0351 & 1.3644 & -0.3521 & 0.5526 \\
1.1314 & -0.3521 & 1.1314 & -0.3521 \\
-0.3521 & 0.5526 & -0.3521 & 0.5526
\end{array}\right] \text {. }
\end{aligned}
$$

Then, according to (14), the dwell time for each subsystem is obtained:

$$
\begin{aligned}
& \text { dwell time for subsystem } 1 \Longrightarrow \tau_{1} \geq 11, \\
& \text { dwell time for subsystem } 2 \Longrightarrow \tau_{2} \geq 10 .
\end{aligned}
$$

To illustrate the simulation results of the FD objective, two cases which include the fault for subsystem 1 and subsystem 2, respectively, are considered. Furthermore, the switching signal is generated by satisfying (31) as shown in Figure 1. The threshold can be determined as $J_{\text {th }}=0.1842$.

Case 1. The fault for subsystem 1 is simulated as a pulse signal with amplitude 1 that occurred from 90 to 120 steps. The generated residual signal $r(k)$ and evaluation of residual evaluation function $J_{r(k)}$ are shown in Figure 2. The simulation results show that when the fault for subsystem 1 occurs, the subsystem 1 is not activated. Since subsystem 1 is activated at 93 steps the residual signal varies sharply, and $J_{r(t)}>J_{\text {th }}$ at 99 steps, which means that the fault for subsystem 1 can be detected 6 steps after subsystem 1 is activated. Hence, the fault for subsystem 1 can be detected.

Case 2. The fault for subsystem 2 is simulated as a pulse signal with amplitude 1 that occurred from 170 to 220 steps. The generated residual signal $r(k)$ and evaluation of residual evaluation function $J_{r(k)}$ are shown in Figure 3. It can be seen that when the fault for subsystem 2 occurs at 170 steps, the residual signal is changed sharply and $J_{r(k)}>J_{\text {th }}$ at 182 steps. Thus, the fault for subsystem 2 can be detected.

From Cases 1 and 2, we see that both faults for subsystem 1 and subsystem 2, respectively, can be detected, and they demonstrate the effectiveness of the proposed design method.

\section{Conclusion}

In this paper, the problem of FD filter design for discrete-time nonlinear impulsive switched systems has been investigated. Firstly, the weight $l_{2}$ performance and the $H_{-}$performance are presented, and sufficient conditions to characterize given performances have been formulated as the form of LMI. Subsequently, the gains of FD filters are obtained by a multiobjective optimization problem. Finally, the effectiveness of the proposed method for discrete-time nonlinear impulsive switched systems is illustrated by the example.

\section{Appendices}

\section{A. Proof of Lemma 8}

When $k \in\left(k_{i}, k_{i+1}\right]$ and $j$ th subsystem is activated, along the trajectory of nonlinear, impulsive switched system (4) gives

$$
\begin{aligned}
\Delta V_{j}(k)= & \tilde{x}^{T}(k)\left(\mathscr{A}_{j}^{T} \mathscr{P}_{j} \mathscr{A}_{j}-\mathscr{P}_{j}\right) \tilde{x}(k) \\
& +\tilde{x}^{T}(k) \mathscr{A}_{j}^{T} \mathscr{P}_{j} \mathscr{B}_{j 1} d(k) \\
& +d^{T}(k) \mathscr{B}_{j 1}^{T} \mathscr{P}_{j} \mathscr{A}_{j} \tilde{x}(k) \\
& +d^{T}(k) \mathscr{B}_{j 1}^{T} \mathscr{P}_{j} \mathscr{B}_{j 1} d(k) \\
& +\widetilde{\Upsilon}_{j}(k, \tilde{x}(k))^{T} \mathscr{P}_{j} \tilde{\Upsilon}_{j}(k, \tilde{x}(k)) \\
& +2 \widetilde{\Upsilon}_{j}(k, \tilde{x}(k))^{T} \mathscr{P}_{j} \mathscr{A}_{j} \tilde{x}(k) \\
& +2 \widetilde{\Upsilon}_{j}(k, \tilde{x}(k))^{T} \mathscr{P}_{j} \mathscr{B}_{j 1} d(k) .
\end{aligned}
$$




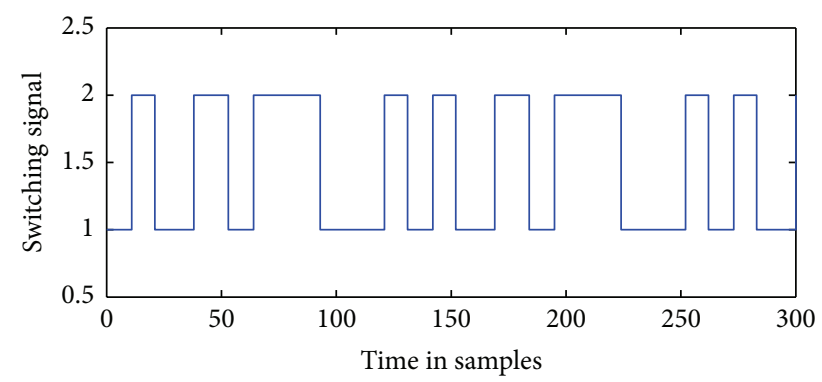

Figure 1: Switching signal.

By Lemma 6, it is clear that

$$
\begin{aligned}
2 \widetilde{\Upsilon}_{j}(k, \tilde{x}(k))^{T} \mathscr{P}_{j} \mathscr{A}_{j} \tilde{x}(k) & \\
\leq & \varepsilon_{j 1} \widetilde{\Upsilon}_{j}(k, \tilde{x}(k))^{T} \widetilde{\Upsilon}_{j}(k, \tilde{x}(k)) \\
& +\varepsilon_{j 1}^{-1} \widetilde{x}^{T}(k) \mathscr{A}_{j}^{T} \mathscr{P}_{j}^{2} \mathscr{A}_{j} \tilde{x}(k), \\
2 \widetilde{\Upsilon}_{j}(k, \tilde{x}(k))^{T} \mathscr{P}_{j} \mathscr{B}_{j 1} d(k) & \\
\leq & \varepsilon_{j 2} \widetilde{\Upsilon}_{j}(k, \tilde{x}(k))^{T} \widetilde{\Upsilon}_{j}(k, \tilde{x}(k)) \\
& +\varepsilon_{j 2}^{-1} d(k)^{T} \mathscr{B}_{j 1}^{T} \mathscr{P}_{j}^{2} \mathscr{B}_{j 1} d(k) .
\end{aligned}
$$

Therefore, when assuming the zero input and using (12), Lemma 7, and Assumption 1, we have the following condition: $\left.\Delta V_{j}\right|_{d(k)=0}(k) \leq \tilde{x}^{T}(k) Q_{a j} \tilde{x}(k)$, where $Q_{a j}=\mathscr{A}_{j}^{T}\left(\mathscr{P}_{j}+\right.$ $\left.\varepsilon_{j 1}^{-1} \mathscr{P}_{j}^{2}\right) \mathscr{A}_{j}-\mathscr{P}_{j}+\left(\varepsilon_{j 1}+\lambda_{j}\right) g_{j} I$. If (13) holds, then $Q_{a j}+\alpha_{j} \mathscr{P}_{j}+$ $\varepsilon_{j 2} g_{j} I \leq 0$, which implies that $Q_{a j} \leq-\alpha_{j} \mathscr{P}_{j}-\varepsilon_{j 2} g_{j} I \leq$ $-\alpha_{j} \mathscr{P}_{j} \leq 0$. Thus, we have

$$
\left.V_{j}(k+1)\right|_{d(k)=0} \leq(1-\alpha) V_{j}(k) .
$$

At the impulsive switching time point $k_{i}$, it has

$$
\begin{aligned}
V_{j}\left(k_{i}^{+}\right) & =\left(\Delta \widetilde{x}\left(k_{i}\right)+\tilde{x}\left(k_{i}\right)\right)^{T} \mathscr{P}_{j}\left(\Delta \tilde{x}\left(k_{i}\right)+\tilde{x}\left(k_{i}\right)\right) \\
& =\left[\left(\mathscr{H}_{j}+I\right) \tilde{x}\left(k_{i}\right)\right]^{T} \mathscr{P}_{j}\left[\left(\mathscr{H}_{j}+I\right) \tilde{x}\left(k_{i}\right)\right]
\end{aligned}
$$

$$
\begin{aligned}
& +2 \widetilde{\Omega}_{j}^{T}\left(k_{i}, \tilde{x}\left(k_{i}\right)\right) \mathscr{P}_{j}\left[\left(\mathscr{H}_{j}+I\right) \tilde{x}\left(k_{i}\right)\right] \\
& +\widetilde{\Omega}_{j}^{T}\left(k_{i}, \tilde{x}\left(k_{i}\right)\right) \mathscr{P}_{j} \widetilde{\Omega}_{j}\left(k_{i}, \tilde{x}\left(k_{i}\right)\right) .
\end{aligned}
$$

By Lemmas 6 and 7 and Assumption 2, it has

$$
\begin{aligned}
& V_{j}\left(k_{i}^{+}\right) \leq\left(\varepsilon_{j 3}+1\right)\left[\left(\mathscr{H}_{j}+I\right) \tilde{x}\left(k_{i}\right)\right]^{T} \\
& . \mathscr{P}_{j}\left[\left(\mathscr{H}_{j}+I\right) \tilde{x}\left(k_{i}\right)\right]+\left(\varepsilon_{j 3}^{-1}+1\right) \widetilde{\Omega}_{j}^{T}\left(k_{i}, \tilde{x}\left(k_{i}\right)\right) \\
& . \mathscr{P}_{j} \widetilde{\Omega}_{j}\left(k_{i}, \tilde{x}\left(k_{i}\right)\right) \\
& \quad \leq\left\{\left(\varepsilon_{j 3}+1\right) \lambda_{\max }\left\{\left(\mathscr{H}_{j}+I\right)^{T} \mathscr{P}_{j}\left(\mathscr{H}_{j}+I\right)\right\}\right. \\
& \left.+\left(\varepsilon_{j 3}^{-1}+1\right) \rho_{j}^{2} \lambda_{\max }\left\{\mathscr{P}_{j}\right\}\right\} \tilde{x}^{T}\left(k_{i}\right) \tilde{x}\left(k_{i}\right) \leq\left.\mu_{p j}\right|_{i} \\
& \cdot V_{j}\left(k_{i}\right),
\end{aligned}
$$

where $\left.\mu_{p j}\right|_{i}=\left(\left(\varepsilon_{j 3}+1\right) \lambda_{\max }\left\{\left(\mathscr{H}_{j}+I\right)^{T} \mathscr{P}_{j}\left(\mathscr{H}_{j}+I\right)\right\}+\left(\varepsilon_{j 3}^{-1}+\right.\right.$ 1) $\left.\rho_{h}^{2} \lambda_{\max }\left\{\mathscr{P}_{j}\right\}\right) / \lambda_{\min }\left\{\mathscr{P}_{p}\right\}$. Therefore, from (A.3) and (A.5), we have

$$
\begin{aligned}
V_{j}(k) & \leq(1-\alpha)^{k-k_{i}} V_{j}\left(k_{i}^{+}\right) \\
& \leq\left.\mu_{p j}\right|_{i}(1-\alpha)^{k-k_{i}} V_{p}\left(k_{i}\right) \leq \cdots \\
& \leq\left.\prod_{\substack{l=1, s \neq q \\
s \in \mathcal{N}, q \in \mathcal{N}}}^{i} \mu_{s q}\right|_{l}(1-\alpha)^{k-k_{0}} V_{j_{0}}\left(k_{0}\right) .
\end{aligned}
$$

Since (14) holds, that is, there exists $\left.\mu_{p j}\right|_{i}>0$ such that $\ln \left(\left.\mu_{p j}\right|_{i}\right)+\left(k_{i}-k_{i-1}\right) \ln (1-\alpha)<0$, then

$$
\left.\prod_{\substack{l=1, s \neq q \\ s \in \mathcal{N}, q \in \mathcal{N}}}^{i} \mu_{s q}\right|_{l}(1-\alpha)^{k_{l}-k_{l-1}}<1 .
$$

Therefore, we conclude that $V_{j}(k)$ converges to zero as $k \rightarrow \infty$; then nonlinear impulsive switched system (11) with $d(k)=0$ is stable.

For any nonzero $d(k) \in l_{2}[0, \infty)$ and zero initial condition $\tilde{x}\left(k_{0}\right)$. Let $\Gamma(k)=r^{T}(k) r(k)-\gamma^{2} d^{T}(k) d(k)$, we can have

$$
\begin{aligned}
\Delta V_{j}(k)+\alpha V_{j}(k)+\Gamma(k) \leq & {\left[\begin{array}{l}
\tilde{x}(k) \\
d(k)
\end{array}\right]^{T} } \\
& \cdot\left[\begin{array}{cc}
\mathscr{Q}_{a j}+\alpha \mathscr{P}_{j}+\varepsilon_{j 2} g_{j} I+\mathscr{C}_{j}^{T} \mathscr{C}_{j} & \mathscr{A}_{j}^{T} \mathscr{P}_{j} \mathscr{B}_{j 1}+\mathscr{C}_{j}^{T} \mathscr{D}_{j 1} \\
* & -\gamma^{2} I+\mathscr{B}_{j 1}^{T}\left(\mathscr{P}_{j}+\varepsilon_{j 2}^{-1} \mathscr{P}_{j}^{2}\right) \mathscr{B}_{j 1}-\mathscr{D}_{j 1}^{T} \mathscr{D}_{j 1}
\end{array}\right]\left[\begin{array}{c}
\tilde{x}(k) \\
d(k)
\end{array}\right] .
\end{aligned}
$$



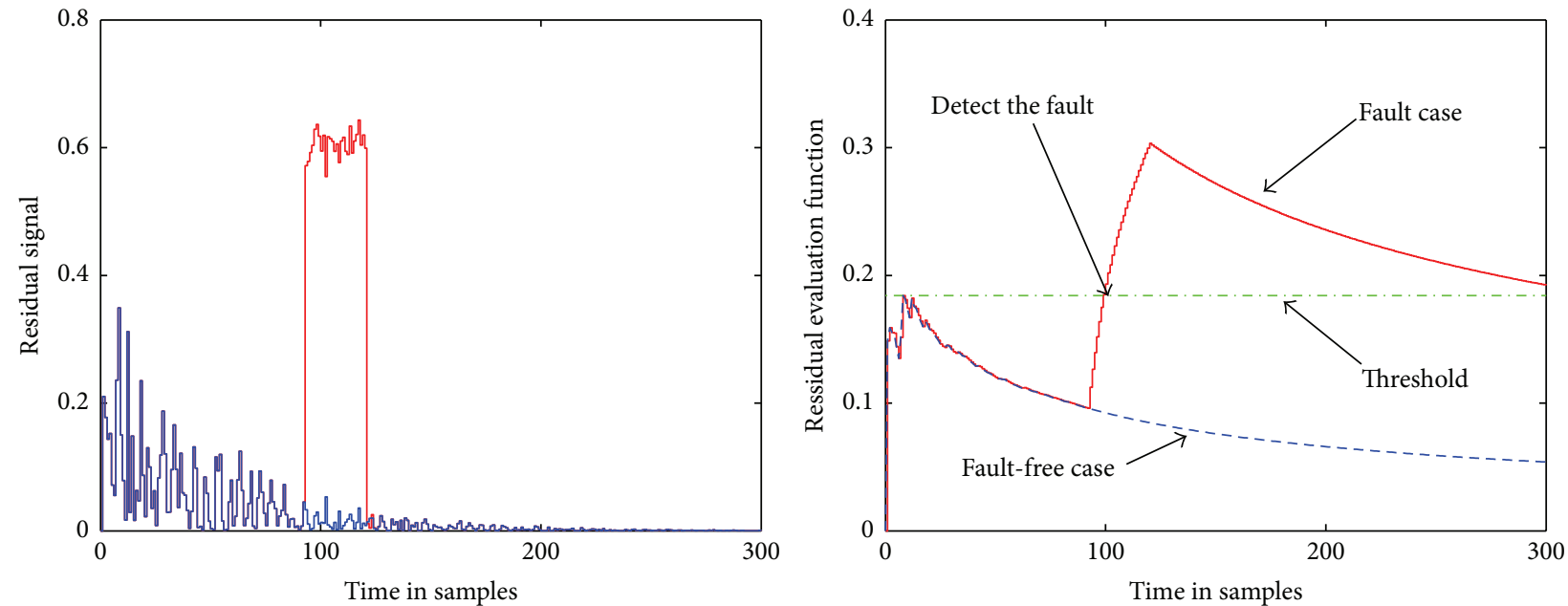

FIgURE 2: Residual signal and residual evaluation function for (Case 1).
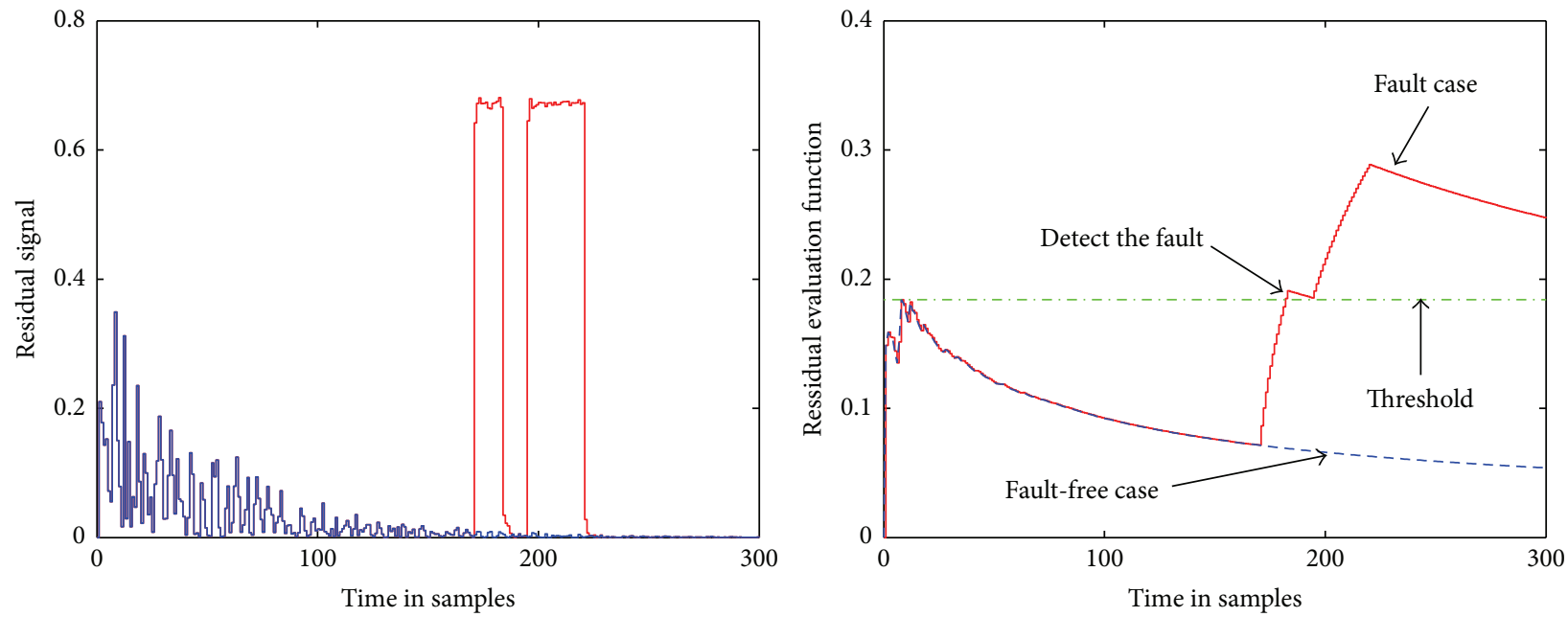

Figure 3: Residual signal and residual evaluation function for (Case 2).

If (13) holds, it is equivalent to $\Delta V_{j}(k)+\alpha V_{j}(k)+\Gamma(k) \leq 0$. For $k \in\left(k_{i}, k_{i+1}\right]$, it can have the following inequality as the similar way:

$$
\begin{aligned}
V_{j}(k) & \\
& \leq\left.\prod_{\substack{l=1, s \neq q \\
s \in \mathcal{N}, q \in \mathcal{N}}}^{i} \mu_{s q}\right|_{l}(1-\alpha)^{k-k_{0}} V_{j_{0}}\left(k_{0}\right) \\
& -\sum_{h=k_{0}}^{k-1}\left\{\left.\prod_{\substack{l=g \|_{h \in\left[g^{\prime}, k_{g+1}\right),} \\
s \in \mathcal{N}, q \in \mathcal{N}, s \neq q}}^{i} \mu_{s q}\right|_{l}\right\}(1-\alpha)^{k-h-1} \Gamma(h) .
\end{aligned}
$$

Under the zero initial condition, it implies that

$$
\sum_{h=k_{0}}^{k-1}\left\{\left.\prod_{\substack{l=g \|_{h \in\left[k_{g}, k_{g+1}\right)}, s \in \mathcal{N}, q \in \mathcal{N}, s \neq q}}^{i} \mu_{s q}\right|_{l}\right\}(1-\alpha)^{k-h-1} \Gamma(h)<0 .
$$

Multiplying both sides of (A.10) by $\left.\prod_{\substack{s \in \mathcal{N}, q \in \mathcal{N}, s \neq q \\ l=1}}^{i} \mu_{s q}\right|_{l} ^{-1}$, one can obtain

$$
\begin{aligned}
& \left.\sum_{h=k_{0}}^{k-1} \prod_{\substack{l=1 \\
s \in \mathcal{N}, q \in \mathcal{N}, s \neq q}}^{g \|_{h \in\left[g_{g}, k_{g+1}\right)}} \mu_{s q}\right|_{l} ^{-1}(1-\alpha)^{k-h-1} r^{T}(k) r(k) \\
& \leq\left.\sum_{h=k_{0}}^{k-1} \prod_{\substack{l=1 \\
s \in \mathcal{N}, q \in \mathcal{N}, s \neq q}}^{g \|_{h \in\left[k_{g}, k_{g+1}\right)}} \mu_{s q}\right|_{l} ^{-1}(1-\alpha)^{k-h-1} \gamma^{2} d^{T}(k) d(k) .
\end{aligned}
$$


Moreover, it follows from the switching sinal that $\left.\prod_{\substack{s \in \mathcal{N}, q \in \mathcal{N}, s \neq q \\ l \|_{h \in\left[k_{g}, k_{g+1}\right)}}} \mu_{s q}\right|_{l} ^{-1} \geq(1-\alpha)^{h-k_{0}}$. Then

$$
\begin{aligned}
& \sum_{h=k_{0}}^{k-1}(1-\alpha)^{k-1-k_{0}} r(s)^{T} r(s) \\
& \leq\left.\sum_{h=k_{0}}^{k-1} \prod_{\substack{l=1 \\
s \in \mathcal{N}, q \in \mathcal{N}, s \neq q}}^{g \|_{h \in\left[k_{g}, k_{g+1}\right)}} \mu_{s q}\right|_{l} ^{-1}(1-\alpha)^{k-h-1} r^{T}(k) r(k) \\
& \leq\left.\sum_{h=k_{0}}^{k-1} \prod_{\substack{l=1 \\
s \in \mathcal{N}, q \in \mathcal{N}, s \neq q}}^{g \|_{h \in\left[k_{g}, k_{g+1}\right)}} \mu_{s q}\right|_{l} ^{-1}(1-\alpha)^{k-h-1} \gamma^{2} d^{T}(k) d(k) \\
& \leq \sum_{h=k_{0}}^{k-1} \gamma^{2} d^{T}(k) d(k) .
\end{aligned}
$$

It further implies that

$$
\sum_{k=k_{0}}^{\infty}(1-\alpha)^{k-k_{0}} r(k)^{T} r(k) \leq \sum_{h=k_{0}}^{\infty} \gamma^{2} d^{T}(k) d(k) .
$$

Therefore, we conclude that discrete-time nonlinear impulsive switched system (11) has the weighted $l_{2}$ performance for any switching signal satisfying (14), which completes the proof.

\section{B. Proof of Theorem 9}

Suppose that (17) holds, and partitioning $\widehat{A}_{f j}=\mathscr{P}_{j 2} A_{f j}$, $\widehat{B}_{f j}=\mathscr{P}_{j 2} B_{f j}, \widehat{C}_{f j}=R_{j}^{T} C_{f j}$, and $\widehat{D}_{f j}=R_{j}^{T} D_{f j},(17)$ means

$$
\begin{gathered}
{\left[\begin{array}{cccccc}
-\bar{\alpha} \mathscr{P}_{j}+\varphi_{a j} I & 0 & \mathscr{A}_{j}^{T} \mathscr{P}_{j} & \mathscr{A}_{j}^{T} \mathscr{P}_{j} & 0 & \mathscr{C}_{j}^{T} R_{j} \\
* & -\gamma^{2} I & \mathscr{B}_{j 1}^{T} \mathscr{P}_{j} & 0 & \mathscr{B}_{j 1}^{T} \mathscr{P}_{j} & \mathscr{D}_{j 1}^{T} R_{j} \\
* & * & -\mathscr{P}_{j} & 0 & 0 & 0 \\
* & * & * & -\varepsilon_{j 1} I & 0 & 0 \\
* & * & * & * & -\varepsilon_{j 2} I & 0 \\
* & * & * & * & 0 & I-\operatorname{He}\left(R_{j}\right)
\end{array}\right]} \\
<0 ;
\end{gathered}
$$

then it is easy to see from (B.1) that $\left(R_{j}-I\right)^{T}\left(R_{j}-I\right) \geq 0$, which implies $I-\operatorname{He}\left(R_{j}\right) \geq-R_{j}^{T} R_{j}$. Then (17) is transformed to

$$
\left[\begin{array}{cccccc}
-\bar{\alpha} \mathscr{P}_{j}+\varphi_{a j} I & 0 & \mathscr{A}_{j}^{T} \mathscr{P}_{j} & \mathscr{A}_{j}^{T} \mathscr{P}_{j} & 0 & \mathscr{C}_{j}^{T} R_{j} \\
* & -\gamma^{2} I & \mathscr{B}_{j 1}^{T} \mathscr{P}_{j} & 0 & \mathscr{B}_{j 1}^{T} \mathscr{P}_{j} & \mathscr{D}_{j 1}^{T} R_{j} \\
* & * & -\mathscr{P}_{j} & 0 & 0 & 0 \\
* & * & * & -\varepsilon_{j 1} I & 0 & 0 \\
* & * & * & * & -\varepsilon_{j 2} I & 0 \\
* & * & * & * & 0 & -R_{j}^{T} R_{j}
\end{array}\right]
$$

$$
<0 \text {. }
$$

Premultiplying $\operatorname{diag}\left\{I, I, \mathscr{P}_{j}^{-1}, I, I, R_{j}^{-T}\right\}$ and postmultiplying $\operatorname{diag}\left\{I, I, \mathscr{P}_{j}^{-1}, I, I, R_{j}^{-1}\right\}$ to (B.2), it is transformed into

$$
\left[\begin{array}{cccccc}
-\bar{\alpha} \mathscr{P}_{j}+\varphi_{a j} I & 0 & \mathscr{A}_{j}^{T} & \mathscr{A}_{j}^{T} \mathscr{P}_{j} & 0 & \mathscr{C}_{j}^{T} \\
* & -\gamma^{2} I & \mathscr{B}_{j 1}^{T} & 0 & \mathscr{B}_{j 1}^{T} \mathscr{P}_{j} & \mathscr{D}_{j 1}^{T} \\
* & * & -\mathscr{P}_{j}^{-1} & 0 & 0 & 0 \\
* & * & * & -\varepsilon_{j 1} I & 0 & 0 \\
* & * & * & * & -\varepsilon_{j 2} I & 0 \\
* & * & * & * & 0 & -I
\end{array}\right]
$$

$<0$,

Then, by using the Schur complement formula, we can see that (B.3) is equivalent to (13). Then, according to Lemma 8, if the conditions (16), (17) hold, the switched system (11) is asymptotically stable with a weighted $l_{2}$ performance for any switching signal satisfying (14), which completes the proof.

\section{Proof of Lemma 10}

Following the same lines as that for Lemma 8, the switched system (19) satisfying switching signal (14) is stable. Then, the $H_{-}$performance defined in (6) for discrete-time nonlinear impulsive switched system (19) is established.

For any nonzero $f(k) \in l_{2}\left[k_{0}, \infty\right)$, consider the following index: $J(k)=\beta^{2} f^{T}(k) f(k)-r^{T}(k) r(k)$. It has the following:

$$
\begin{aligned}
\Delta V_{j}(k)+\alpha V_{j}(k)+J(k) \leq & {\left[\begin{array}{c}
\tilde{x}(k) \\
f(k)
\end{array}\right]^{T} } \\
& \cdot\left[\begin{array}{cc}
\mathscr{Q}_{b j}+\alpha \mathscr{P}_{j}+\varepsilon_{j 4} \mathcal{g}_{j} I-\mathscr{C}_{j}^{T} \mathscr{C}_{j} & \mathscr{B}_{j 2}^{T} \mathscr{P}_{j} \mathscr{A}_{j}-\mathscr{C}_{j}^{T} \mathscr{D}_{j 2} \\
* & \beta^{2} I+\mathscr{B}_{j 2}^{T}\left(\mathscr{P}_{j}+\varepsilon_{j 4}^{-1} \mathscr{P}_{j}^{2}\right) \mathscr{B}_{j 2}+\mathscr{D}_{j 2}^{T} \mathscr{D}_{j 2}
\end{array}\right]\left[\begin{array}{c}
\tilde{x}(k) \\
f(k)
\end{array}\right],
\end{aligned}
$$


where $Q_{b j}=Q_{a j}$. If (21) holds, it is equivalent to $\Delta V_{j}(k)+$ $\alpha V_{j}(k)+J(k) \leq 0$. By iteration operation on the above inequality for $k \in\left(k_{i}, k_{i+1}\right]$, we have

$$
\begin{aligned}
V_{j}(k) & \\
\leq & \left.\prod_{\substack{l=1, s \neq q \\
s \in \mathcal{N}, q \in \mathcal{N}}}^{i} \mu_{s q}\right|_{l}(1-\alpha)^{k-k_{0}} V_{j_{0}}\left(k_{0}\right) \\
& -\sum_{h=k_{0}}^{k-1}\left\{\left.\prod_{\substack{l=g \|_{h \in\left[k_{g}, k_{g+1}\right)}, s \in \mathcal{N}, q \in \mathcal{N}, s \neq q}}^{i} \mu_{s q}\right|_{l}\right\}(1-\alpha)^{k-h-1} J(h) .
\end{aligned}
$$

Under the zero initial condition, one has $V_{j}\left(k_{0}\right)=0$ and $V_{j}(k)>0$; thus

$$
\sum_{h=k_{0}}^{k-1}\left\{\left.\prod_{\substack{l=g \|_{h \in\left[k_{g}, k_{g+1}\right)}, s \in \mathcal{N}, q \in \mathcal{N}, s \neq q}}^{i} \mu_{s q}\right|_{l}\right\}(1-\alpha)^{k-h-1} J(h) \leq 0
$$

Thus, from $\left.\mu_{s q}\right|_{l}>1$, we obtain that

$$
\begin{aligned}
& \sum_{h=k_{0}}^{k-1}(1-\alpha)^{k-h-1} J(h) \\
& \quad \leq \sum_{h=k_{0}}^{k-1}\left\{\left.\prod_{\substack{l=g \|_{h \in\left[k_{g}, k_{g+1}\right)}, s \in \mathcal{N}, q \in \mathcal{N}, s \neq q}}^{i} \mu_{s q}\right|_{l}\right\}(1-\alpha)^{k-h-1} J(h) \leq 0 .
\end{aligned}
$$

Then we have

$$
\begin{aligned}
& \sum_{h=k_{0}}^{k-1}(1-\alpha)^{k-h-1} \beta^{2} f^{T}(h) f(h) \\
& \quad \leq \sum_{h=k_{0}}^{k-1}(1-\alpha)^{k-h-1} r^{T}(h) r(h) .
\end{aligned}
$$

When taking $k$ from $k_{0}$ to $\infty$, we can further obtain that

$$
\sum_{h=k_{0}}^{\infty} \beta^{2} f^{T}(h) f(h) \leq \sum_{h=k_{0}}^{\infty} r^{T}(h) r(h) .
$$

Therefore, we conclude that discrete-time nonlinear impulsive switched system (19) has the $H_{-}$performance for any switching signal satisfying (14), which completes the proof.

\section{Proof of Theorem 11}

Denote $\bar{\alpha}=1-\alpha, \varphi_{b j}=\left(\varepsilon_{j 1}+\varepsilon_{j 4}+\lambda_{j}\right) g_{j}$, and
To establish the convex condition, (21) can be rewritten as follows:

$$
\left[\begin{array}{cc}
\mathscr{C}_{j} & \mathscr{D}_{j 2} \\
I & 0 \\
0 & I
\end{array}\right] \mathscr{M}_{b j}\left[\begin{array}{cc}
\mathscr{C}_{j} & \mathscr{D}_{j 2} \\
I & 0 \\
0 & I
\end{array}\right]<0 .
$$

On the other hand,

$$
\left[\begin{array}{lll}
I & 0 & 0
\end{array}\right] \mathscr{M}_{b j}\left[\begin{array}{lll}
I & 0 & 0
\end{array}\right]^{T}=-I<0 .
$$

Based on Projection Lemma, it follows from (D.2) and (D.3) that

$$
\mathscr{M}_{b j}+\operatorname{He}\left(\left[\begin{array}{c}
-I \\
\mathscr{C}_{j}^{T} \\
\mathscr{D}_{j 2}^{T}
\end{array}\right] \mathscr{W}_{b j}\left[\begin{array}{lll}
0 & I & 0 \\
0 & 0 & I
\end{array}\right]\right)<0,
$$

where $\mathscr{W}_{b j}$ introduced by Projection Lemma is the matrix variable of appropriate dimensions. Partition $\mathscr{W}_{b j}$ as $\mathscr{W}_{b j}=$ $\left[\begin{array}{ll}\mathscr{W}_{b j 1} & \mathscr{W}_{b j 2}\end{array}\right]$. By Schur complement, (D.4) is equivalent to 


$$
\left[\begin{array}{cccccc}
-I & -\mathscr{W}_{b j 1} & -\mathscr{W}_{b j 2} & 0 & 0 & 0 \\
* & -\bar{\alpha} \mathscr{P}_{j}+\varphi_{b j} I+\operatorname{He}\left(\mathscr{C}_{j}^{T} \mathscr{W}_{b j 1}\right) & \mathscr{C}_{j}^{T} \mathscr{W}_{b j 2}+\mathscr{W}_{b j 1}^{T} \mathscr{D}_{j 2} & \mathscr{A}_{j}^{T} \mathscr{P}_{j} & \mathscr{A}_{j}^{T} \mathscr{P}_{j} & 0 \\
* & * & \beta^{2} I+\operatorname{He}\left(\mathscr{D}_{j 2}^{T} \mathscr{W}_{b j 2}\right) & \mathscr{B}_{j 2}^{T} \mathscr{P}_{j} & 0 & \mathscr{B}_{j 2}^{T} \mathscr{P}_{j} \\
* & * & * & -\mathscr{P}_{j} & 0 & 0 \\
* & * & * & * & -\varepsilon_{j 1} I & 0 \\
* & * & * & * & * & -\varepsilon_{j 3} I
\end{array}\right]<0 .
$$

Let $\mathscr{W}_{b j 1}=\left[\begin{array}{ll}\mathscr{R}_{j} & \mathscr{R}_{j}\end{array}\right], \mathscr{W}_{b j 2}=R_{j}$, and $\mathscr{R}_{j}=\left[\begin{array}{ll}R_{j} & 0\end{array}\right]$ and define $\widehat{A}_{f j}=\mathscr{P}_{j 2} A_{f j}, \widehat{B}_{f j}=\mathscr{P}_{j 2} B_{f j}, \widehat{C}_{f j}=R_{j}^{T} C_{f j}, \widehat{D}_{f j}=$ $R_{j}^{T} D_{f j} \widehat{C}_{f f j}=\mathscr{R}_{j}^{T} C_{f j}$, and $\widehat{D}_{f f j}=\mathscr{R}_{j}^{T} D_{f j}$; then (D.5) becomes (17). Hence if the conditions (16) and (17) hold, nonlinear impulsive switched system (19) is stable and guarantees the $H_{-}$performance, which completes the proof.

\section{Conflict of Interests}

The authors declare that there is no conflict of interests regarding the publication of this paper.

\section{Acknowledgments}

This work is supported by the Funds of National Science of China (Grant no. 61403075) and Natural Science Foundation of Jilin Province (Grant no. 20140520060JH).

\section{References}

[1] J. Chen and P. R. Patton, Robust Model-Based Fault Diagnosis for Dynamic Systems, Kluwer Academic Publishers, Boston, Mass, USA, 1999.

[2] I. Hwang, S. Kim, Y. Kim, and C. E. Seah, "A survey of fault detection, isolation, and reconfiguration methods," IEEE Transactions on Control Systems Technology, vol. 18, no. 3, pp. 636-653, 2010.

[3] M. Du and P. Mhaskar, "Isolation and handling of sensor faults in nonlinear systems," Automatica, vol. 50, no. 4, pp. 1066-1074, 2014.

[4] Y. Long and G.-H. Yang, "Fault detection for a class of networked control systems with finite-frequency servo inputs and random packet dropouts," IET Control Theory \& Applications, vol. 6, no. 15, pp. 2397-2408, 2012.

[5] S. He and F. Liu, "Fuzzy model-based fault detection for Markov jump systems," International Journal of Robust and Nonlinear Control, vol. 19, no. 11, pp. 1248-1266, 2009.

[6] Z. H. Li and I. M. Jaimoukha, "Observer-based fault detection and isolation filter design for linear time-invariant systems," International Journal of Control, vol. 82, no. 1, pp. 171-182, 2009.

[7] X.-J. Li and G.-H. Yang, "Fault detection for linear stochastic systems with sensor stuck faults," Optimal Control Applications and Methods, vol. 33, no. 1, pp. 61-80, 2012.

[8] Y. Long and G.-H. Yang, "Fault detection and isolation for networked control systems with finite frequency specifications,"
International Journal of Robust and Nonlinear Control, vol. 24, no. 3, pp. 495-514, 2014.

[9] H. Lin and P. J. Antsaklis, "Stability and stabilizability of switched linear systems: a survey of recent results," IEEE Transactions on Automatic Control, vol. 54, no. 2, pp. 308-322, 2009.

[10] Q. Wang, T. Wang, Y. Hou, and C. Dong, "Sign stability for switched linear systems and its application in flight control," Journal of Applied Mathematics, vol. 2014, Article ID 391617, 9 pages, 2014.

[11] S. Lim and J. P. How, "Modeling and $H_{\infty}$ control for switched linear parameter-varying missile autopilot," IEEE Transactions on Control Systems Technology, vol. 11, no. 6, pp. 830-838, 2003.

[12] M. Barkhordari Yazdi and M. R. Jahed-Motlagh, "Stabilization of a CSTR with two arbitrarily switching modes using modal state feedback linearization," Chemical Engineering Journal, vol. 155, no. 3, pp. 838-843, 2009.

[13] G. S. Deaecto, M. Souza, and J. C. Geromel, "Discrete-time switched linear systems state feedback design with application to networked control," Institute of Electrical and Electronics Engineers. Transactions on Automatic Control, vol. 60, no. 3, pp. 877-881, 2015.

[14] D. S. Du, B. Jiang, P. Shi, and S. S. Zhou, " $H_{\infty}$ filtering of discrete-time switched systems with state delays via switched Lyapunov function approach," IEEE Transactions on Automatic Control, vol. 52, no. 8, pp. 1520-1525, 2007.

[15] Q. Su and J. Zhao, "Stabilization of a class of switched systems with state constraints," Nonlinear Dynamics, vol. 70, no. 2, pp. 1499-1510, 2012.

[16] O. Karabacak, "Dwell time and average dwell time methods based on the cycle ratio of the switching graph," Systems \& Control Letters, vol. 62, no. 11, pp. 1032-1037, 2013.

[17] J. Yao, Z.-H. Guan, G. R. Chen, and D. W. C. Ho, "Stability, robust stabilization and $H_{\infty}$ control of singular-impulsive systems via switching control," Systems \& Control Letters, vol. 55 , no. 11, pp. 879-886, 2006.

[18] W. Xiang and J. Xiao, "Stability analysis and control synthesis of switched impulsive systems," International Journal of Robust and Nonlinear Control, vol. 22, no. 13, pp. 1440-1459, 2012.

[19] S. W. Zhao and J. T. Sun, "A geometric approach for reachability and observability of linear switched impulsive systems," Nonlinear Analysis: Theory, Methods \& Applications, vol. 72, no. 11, pp. 4221-4229, 2010.

[20] H. Xu, K. L. Teo, and X. Liu, "Robust stability analysis of guaranteed cost control for impulsive switched systems," IEEE Transactions on Systems, Man, and Cybernetics Part B: Cybernetics, vol. 38, no. 5, pp. 1419-1422, 2008. 
[21] Z. Zhang, "Robust $H_{\infty}$ control of a class of discrete impulsive switched systems," Nonlinear Analysis: Theory, Methods \& Applications, vol. 71, no. 12, pp. e2790-e2796, 2009.

[22] M. Du and P. Mhaskar, "A safe-parking and safe-switching framework for fault-tolerant control of switched nonlinear systems," International Journal of Control, vol. 84, no. 1, pp. 9-23, 2011.

[23] D. Wang, W. Wang, and P. Shi, "Robust fault detection for switched linear systems with state delays," IEEE Transactions on Systems, Man, and Cybernetics, Part B: Cybernetics, vol. 39, no. 3, pp. 800-805, 2009.

[24] D. Du, B. Jiang, and P. Shi, "Fault detection for discrete-time switched systems with intermittent measurements," International Journal of Control, vol. 85, no. 1, pp. 78-87, 2012.

[25] J. Li and G.-H. Yang, "Fault detection filter design for discretetime switched linear systems with mode-dependent average dwell-time," International Journal of Adaptive Control and Signal Processing, vol. 28, no. 1, pp. 77-95, 2014.

[26] D. S. Du, B. Jiang, P. Shi, and H. R. Karimi, "Fault detection for continuous-time switched systems under asynchronous switching," International Journal of Robust and Nonlinear Control, vol. 24, no. 11, pp. 1694-1706, 2014.

[27] H. Xu and K. L. Teo, "Exponential stability with $L_{2}$-gain condition of nonlinear impulsive switched systems," IEEE Transactions on Automatic Control, vol. 55, no. 10, pp. 24292433, 2010.

[28] P. M. Frank and X. Ding, "Survey of robust residual generation and evaluation methods in observer-based fault detection systems," Journal of Process Control, vol. 7, no. 6, pp. 403-424, 1997.

[29] H. Xu, X. Liu, and K. L. Teo, "Robust $H_{\infty}$ stabilisation with definite attenuance of an uncertain impulsive switche system," The ANZIAM Journal, vol. 46, no. 4, pp. 471-484, 2005. 


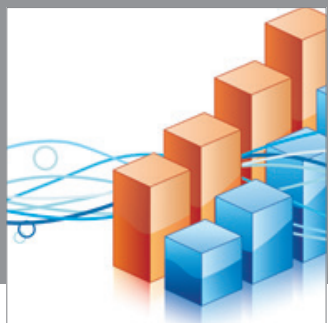

Advances in

Operations Research

mansans

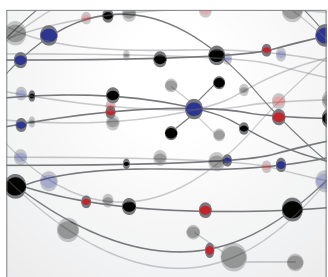

The Scientific World Journal
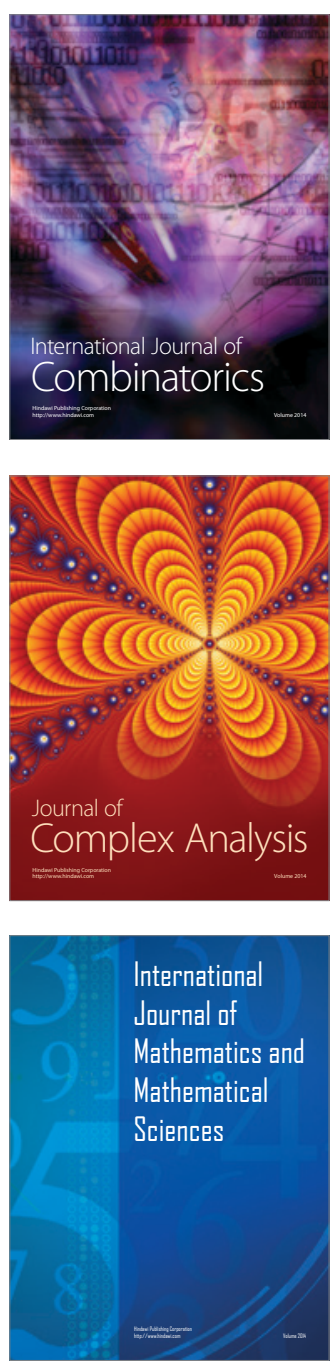
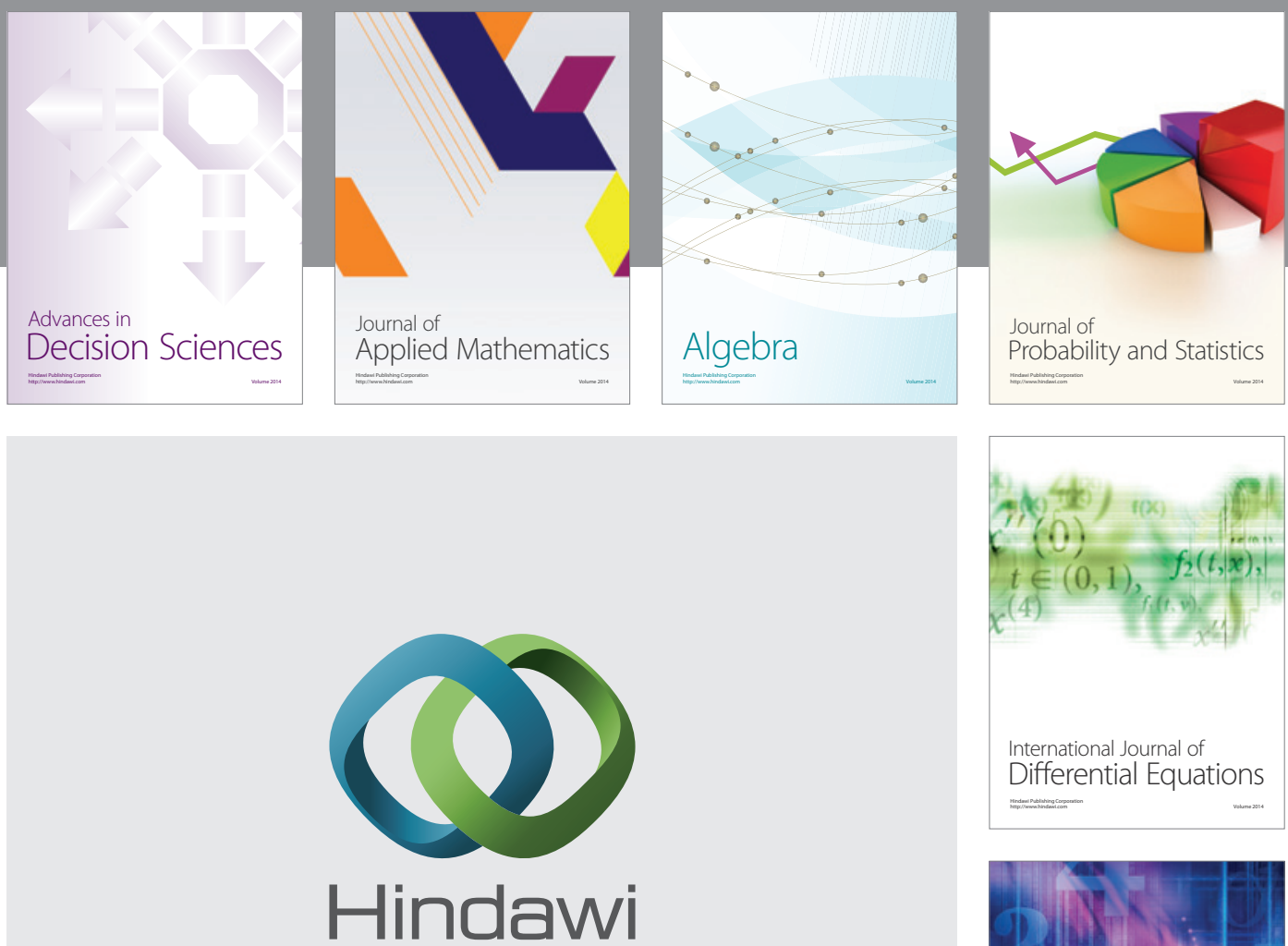

Submit your manuscripts at http://www.hindawi.com
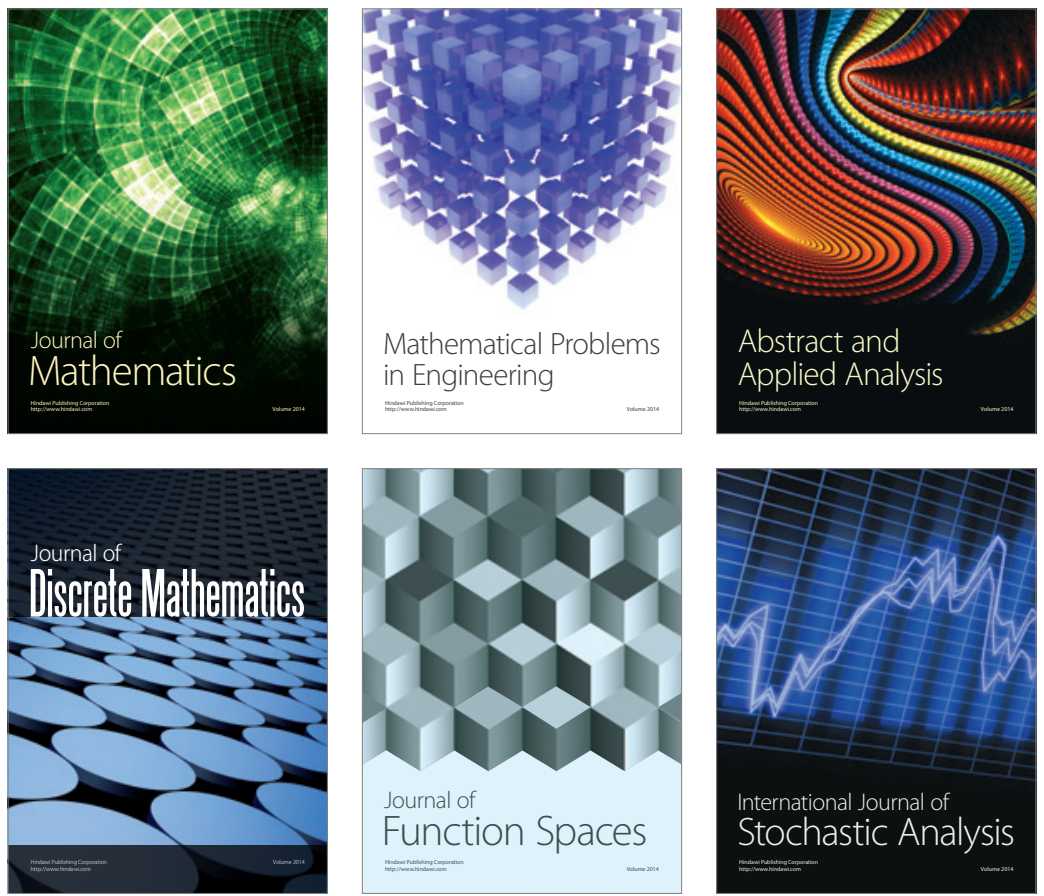

Journal of

Function Spaces

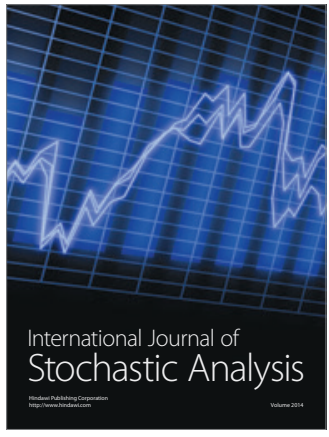

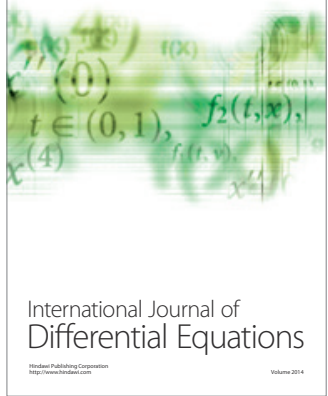
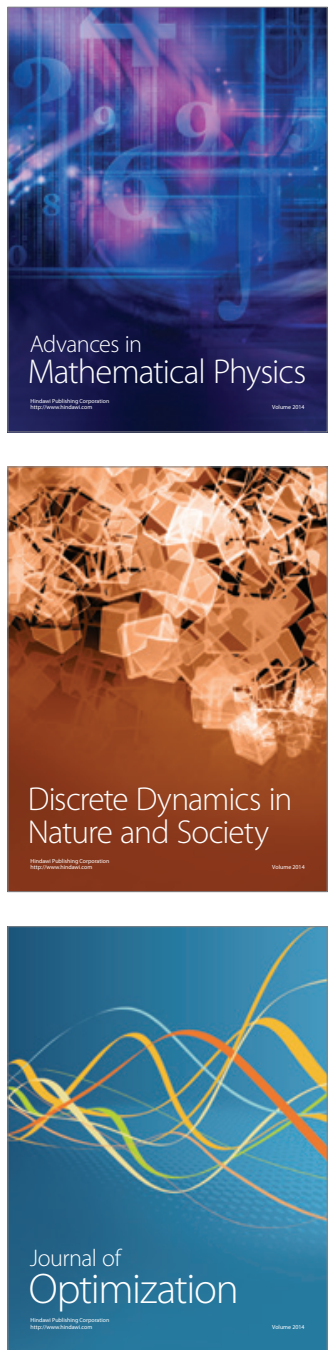